\title{
On the absolute continuity of one-dimensional SDE's driven by a fractional Brownian motion
}

\author{
Ivan Nourdin \\ Université Henri Poincaré, Institut de Mathématiques Élie Cartan, B.P. 239 \\ 54506 Vandouvre-lès-Nancy Cédex, France \\ Ivan. Nourdin@iecn.u-nancy.fr \\ Thomas Simon \\ Université d'Évry-Val d'Essonne, Équipe d'Analyse et Probabilités \\ Boulevard François Mitterand, 91025 Évry Cédex, France \\ Thomas.Simon@maths . univ-evry.fr
}

\begin{abstract}
The problem of absolute continuity for a class of SDE's driven by a real fractional Brownian motion of any Hurst index is adressed. First, we give an elementary proof of the fact that the solution to the SDE has a positive density for all $t>0$ when the diffusion coefficient does not vanish, echoing in the fractional Brownian framework the main result we had previously obtained for Marcus equations driven by Lévy processes [9]. Second, we extend in our setting the classical entrance-time criterion of Bouleau-Hirsch[2].
\end{abstract}

Keywords: Absolute continuity - Doss-Sussmann transformation - Fractional Brownian motion - Newton-Côtes SDE.

MSC 2000: 60G18, 60H10.

\section{Introduction}

In this note we study the absolute continuity of the solutions at any time $t>0$ to SDE's of the type:

$$
X_{t}=x_{0}+\int_{0}^{t} b\left(X_{s}\right) d s+\int_{0}^{t} \sigma\left(X_{s}\right) \diamond d B_{s}^{H},
$$

where $b, \sigma$ are real functions and $B^{H}$ is a linear fractional Brownian motion (fBm) with Hurst index $H \in(0,1)$. In $(1), \diamond$ means a particular type of linear non-semimartingale integrators, the so-called Newton-Côtes integrator, which was recently introduced by one of us et al. [7] [8]. Roughly speaking, $\diamond$ is an operator defined through a limiting procedure involving the usual Newton-Côtes linear approximator (whose order depends on the roughness of the path $B^{H}$ ), and a forward-backward decomposition à la Russo-Vallois [12]. This gives a reasonable class of solutions to (1) as soon as $\sigma$ is regular enough. We refer to [7] and [8] for more details on this topic.

The main interest of $\diamond$ is that it yields a first order Itô's formula: if $f: \mathbb{R}^{2} \rightarrow \mathbb{R}$ is regular enough and $Y: \Omega \times \mathbb{R}^{+} \rightarrow \mathbb{R}$ is a bounded variation process, then for every $t \geq 0$

$$
f\left(B_{t}^{H}, Y_{t}\right)=f\left(0, Y_{0}\right)+\int_{0}^{t} f_{x}^{\prime}\left(B_{s}^{H}, Y_{s}\right) \diamond d B_{s}^{H}+\int_{0}^{t} f_{y}^{\prime}\left(B_{s}^{H}, Y_{s}\right) d Y_{s},
$$


see [8] for details. This formula allows to solve (1) through Doss [5] and Sussmann [13]'s classical computations. More precisely, our solution $X$ is given by $X_{t}=\varphi\left(B_{t}^{H}, Y_{t}\right)$ for every $t>0$ where $(x, y) \mapsto \varphi(x, y)$ is the flow associated to $\sigma$ :

$$
\varphi_{x}^{\prime}(x, y)=\sigma(\varphi(x, y)), \varphi(0, y)=y \text { for every }(x, y) \in \mathbb{R}^{2},
$$

and $Y$ is the solution to the random ODE

$$
Y_{t}=x_{0}+\int_{0}^{t} a\left(B_{s}^{H}, Y_{s}\right) d s
$$

with the notation

$$
a(x, y)=\frac{b(\varphi(x, y))}{\varphi_{y}^{\prime}(x, y)}=b(\varphi(x, y)) \exp \left\{-\int_{0}^{x} \sigma^{\prime}(\varphi(u, y)) d u\right\}
$$

for every $(x, y) \in \mathbb{R}^{2}$. In the sequel, we will only refer to $X$ as given by the above DossSussmann transformation and we will study the absolute continuity with respect to the Lebesgue measure of $X_{t}$ for any $t>0$.

Our first result, which is given in Section 2, states that $X_{t}$ has a positive density for every $t>0$ as soon as $\sigma$ does not vanish. Notice that in the much more difficult framework where the driving process of (1) is a non Gaussian Lévy process with infinitely many jumps, the same criterion was obtained in [9]. Here, the simple proof relies on a suitable Girsanov transformation [11] which reduces to the easy case when $b \equiv 0$, i.e. when $X_{t}=\varphi\left(B_{t}^{H}, x_{0}\right)$ for every $t>0$. This positivity result is related to Proposition 6 in [1], where in a multidimensional setting but without drift, a sufficient condition (which becomes $\sigma\left(x_{0}\right) \neq 0$ in dimension one) under which $X_{t}$ has a density for every $t>0$ was given, as well as an equivalent of the density $f_{t}$ at $x_{0}$ when $t \rightarrow 0$. We remark that in dimension one, a closed formula - see (5) below - can be readily obtained.

Of course, this non-vanishing condition on $\sigma$ is not optimal. For instance, thinking of the equation $d X_{t}=X_{t} \diamond d B_{t}^{H}$ whose solution is $X_{t}=X_{0} \exp B_{t}^{H}$, we see that the positivity assumption on $\sigma$ is not necessary. Moreover, in the Brownian case $H=1 / 2$, it is well-known that this criterion can be relaxed either into a condition of Hörmander type when $\sigma$ is regular enough - see e.g. [10] p. 111, or into an optimal criterion involving the entrance time into $\{\sigma(x) \neq 0\}$ when $\sigma$ has little regularity - see Theorem 6.3. in [2]. We did not try to go in the Hörmander direction, since the computations involving Newton-Côtes integrals become quite messy. Nevertheless, we were able to obtain a literal extension of Bouleau-Hirsch's criterion for any $H \in(0,1)$. This extension may seem a little surprising, since Bouleau-Hirsch's criterion bears a Markovian flavour, whereas the solution to our $\mathrm{SDE}$ is not Markovian in general. The proof, which is given in Section 3, consists in computing the Malliavin derivative of $X_{t}$ via the Doss-Sussmann transformation, and then using a general non-degeneracy criterion of Nualart-Zakai.

Notice finally that the computation of this Malliavin derivative relies mainly on the existence of a Stratonovich change of variable formula. Hence, our Theorem B below could probably be extended to other type of "rough" equations driven by fBm, see e.g. [4] and [6]. In these two papers there are restrictions from below on the Hurst parameter of the driving $\mathrm{fBm}$, but on the other hand this latter is allowed to be multidimensional. Since BouleauHirsch's criterion also works in a multidimensional framework (with a more complicated formulation for the entrance-time), one may ask for a general fractional extension of this result. The present note can be viewed as a first attempt in this direction. 


\section{A non-vanishing criterion on the diffusion coefficient}

The following theorem, whose proof is elementary, yields a first simple criterion on $\sigma$ according to which $X_{t}$ has a positive density on $\mathbb{R}$ for every $t>0$.

Theorem A If $\sigma$ does not vanish, then $X_{t}$ has a positive density on $\mathbb{R}$ for every $t>0$.

Proof. Considering $-B^{H}$ instead of $B^{H}$ if necessary, we may suppose that $\sigma>0$. Recalling that $\varphi: \mathbb{R}^{2} \rightarrow \mathbb{R}$ is the flow associated with $\sigma$, we first notice that for every fixed $y \in \mathbb{R}$, the function $x \mapsto \varphi(x, y)$ is a bijection onto $\mathbb{R}$. Indeed, $\varphi(\cdot, y)$ is clearly increasing and $\ell=\lim _{x \rightarrow+\infty} \varphi(x, y)$ exists in $\mathbb{R} \cup\{+\infty\}$. If $\ell \neq+\infty$, then $\lim _{x \rightarrow \infty} \varphi_{x}^{\prime}(x, y)=\sigma(\ell)>0$ and $\lim _{x \rightarrow+\infty} \varphi(x, y)=+\infty$. Similarly, we can show that $\lim _{x \rightarrow-\infty} \varphi(x, y)=-\infty$, which yields the desired property. We will denote by $\psi: \mathbb{R}^{2} \rightarrow \mathbb{R}$ the inverse of $\varphi$, i.e. $\psi(x, y)$ is the unique solution to $\varphi(\psi(x, y), y)=x$.

(i) When $b \equiv 0$, we have $X_{t}=\varphi\left(B_{t}^{H}, x_{0}\right)$ for every $t>0$ and we can write, for every $A \in \mathcal{B}(\mathbb{R})$,

$$
\begin{aligned}
\mathbb{P}\left(X_{t} \in A\right) & =\mathbb{P}\left(B_{t}^{H} \in \psi\left(A, x_{0}\right)\right)=\frac{1}{\sqrt{2 \pi t^{2 H}}} \int_{\psi\left(A, x_{0}\right)} \mathrm{e}^{-\frac{u^{2}}{2 t^{2 H}}} d u \\
= & \frac{1}{\sqrt{2 \pi t^{2 H}}} \int_{A} \mathrm{e}^{-\frac{\varphi\left(v, x_{0}\right)^{2}}{2 t^{2 H}}}\left|\sigma\left(\varphi\left(v, x_{0}\right)\right)\right| d v .
\end{aligned}
$$

Hence, $X_{t}$ has an explicit positive density given by

$$
f_{X_{t}}(v)=\frac{1}{\sqrt{2 \pi t^{2 H}}} \mathrm{e}^{-\frac{\varphi\left(v, x_{0}\right)^{2}}{2 t^{2 H}}}\left|\sigma\left(\varphi\left(v, x_{0}\right)\right)\right| .
$$

(ii) When $b \not \equiv 0$, we can first suppose that $b$ has compact support, by an immediate approximation argument. Besides, for every $t>0$, we have

$$
X_{t}=\varphi\left(B_{t}^{H}, Y_{t}\right)=\varphi\left(B_{t}^{H}, \varphi\left(\psi\left(Y_{t}, x_{0}\right), x_{0}\right)\right)=\varphi\left(B_{t}^{H}+\psi\left(Y_{t}, x_{0}\right), x_{0}\right),
$$

the last equality coming from the flow property of $\varphi$. Since $b$ has compact support, it is easy to see from (4) and the bijection property of $\varphi$ that $Y_{t}$ is a bounded random variable for every $t>0$. Hence $\psi\left(Y_{t}, x_{0}\right)$ is also bounded for every $t>0$ and we can appeal to Girsanov's theorem for fBm (see Theorem 3.1 in [11]), which yields

$$
X_{t}=\varphi\left(\tilde{B}_{t}^{H}, x_{0}\right),
$$

where $\tilde{M}$ is a fBm under a probability $\mathbb{Q}$ equivalent to $\mathbb{P}$. Hence we are reduced to the case $b \equiv 0$ and we can conclude from above that, under $\mathbb{Q}, X_{t}$ has a positive density over $\mathbb{R}$. Since $\mathbb{P}$ and $\mathbb{Q}$ are equivalent, the same holds under $\mathbb{P}$.

Remark Theorem A entails in particular that $\operatorname{Supp} \mathrm{X}_{\mathrm{t}}=\mathbb{R}$ for every $t>0$. Actually, this support property can be extended on the functional level: when $\sigma$ does not vanish, it follows easily from Doss's arguments [5] that $\operatorname{Supp} X=\mathcal{C}_{x_{0}}$, where $X=\left\{X_{t}, t \geq 0\right\}$ is viewed as a random variable valued in $\mathcal{C}_{x_{0}}$, the set of continuous functions from $\mathbb{R}^{+}$to $\mathbb{R}$ starting from $x_{0}$ endowed with the local supremum norm. 


\section{Extension of a result of Bouleau-Hirsch}

In this section we extend Theorem A quite considerably, giving a necessary and sufficient condition on $\sigma$ in the spirit of Bouleau-Hirsch's [2] criterion. However our arguments are somewhat more elaborate, and we first need to recall a few facts about the Gaussian analysis related to fractional Brownian motion. In order to simplify the presentation and without loss of generality, we will fix an horizon $T>0$ to (1), hence we will define fBm on $[0, T]$ only.

\subsection{Some recalls about fractional Brownian Motion}

Let us give a few facts about the Gaussian structure of fBm and its Malliavin derivative process, following Sect. 3.1 in [11] and Chap. 1.2 in [10]. Set

$$
R_{H}(t, s):=\frac{1}{2}\left(s^{2 H}+t^{2 H}-|s-t|^{2 H}\right), s, t \in[0, T] .
$$

Let $\mathcal{E}$ be the set of step-functions on $[0, T]$. Consider the Hilbert space $\mathcal{H}$ defined as the closure of $\mathcal{E}$ wih respect to the scalar product

$$
\left(\mathbf{1}_{[0, t]}, \mathbf{1}_{[0, s]}\right)_{\mathcal{H}}=R_{H}(t, s) .
$$

More precisely, if we set

$$
K_{H}(t, s)=\Gamma(H+1 / 2)^{-1}(t-s)^{H-1 / 2} F(H-1 / 2,1 / 2-H ; H+1 / 2,1-t / s),
$$

where $F$ stands for the standard hypergeometric function, and define the linear operator $K_{H}^{*}$ from $\mathcal{E}$ to $L^{2}([0, T])$ by

$$
\left(K_{H}^{*} \varphi\right)(s)=K_{H}(T, s) \varphi(s)+\int_{s}^{T}(\varphi(r)-\varphi(s)) \frac{\partial K_{H}}{\partial r}(r, s) d r,
$$

then $\mathcal{H}$ is isometric to $L^{2}([0, T])$ thanks to the equality

$$
(\varphi, \rho)_{\mathcal{H}}=\int_{0}^{T}\left(K_{H}^{*} \varphi\right)(s)\left(K_{H}^{*} \rho\right)(s) d s .
$$

$B^{H}$ is a centred Gaussian process with covariance function $R_{H}(t, s)$, hence its associated Gaussian space is isometric to $\mathcal{H}$ through the mapping $\mathbf{1}_{[0, t]} \mapsto B_{t}^{H}$.

Let $f: \mathbb{R}^{n} \rightarrow \mathbb{R}$ be a smooth function with compact support and consider the random variable $F=f\left(B_{t_{1}}^{H}, \ldots, B_{t_{n}}^{H}\right)$ (we then say that $F$ is a smooth random variable). The derivative process of $F$ is the element of $L^{2}(\Omega, \mathcal{H})$ defined by

$$
D_{s} F=\sum_{i=1}^{n} \frac{\partial f}{\partial x_{i}}\left(B_{t_{1}}^{H}, \ldots, B_{t_{n}}^{H}\right) \mathbf{1}_{\left[0, t_{i}\right]}(s) .
$$

In particular $D_{s} B_{t}^{H}=\mathbf{1}_{[0, t]}(s)$. As usual, $\mathbb{D}^{1,1}$ is the closure of smooth random variables with respect to the norm

$$
\|F\|_{1,1}=\mathbb{E}[|F|]+\mathbb{E}\left[\|D \cdot F\|_{\mathcal{H}}\right]
$$

and $\mathbb{D}_{\text {loc }}^{1,1}$ is it associated local domain, that is the set of random variables $F$ such that there exists a sequence $\left\{\left(\Omega_{n}, F_{n}\right), n \geq 1\right\} \subset \mathcal{F} \times \mathbb{D}^{1,1}$ such that $\Omega_{n} \uparrow \Omega$ a.s. and $F=F_{n}$ a.s. on $\Omega_{n}$ (see [10] p. 45 for more details). We finally recall the following criterion which is due to Nualart-Zakai (see Theorem 2.1.3 in [10]) :

Theorem 1 (Nualart-Zakai) If $F \in \mathbb{D}_{\text {loc }}^{1,1}$ and a.s. $\|D . F\|_{\mathcal{H}}>0$, then $F$ has a density with respect to Lebesgue measure on $\mathbb{R}$. 


\subsection{Statement and proof of the main result}

Let $J=\sigma^{-1}(\{0\})$ and int $J$ be the interior of $J$. Consider the deterministic equation

$$
x_{t}=x_{0}+\int_{0}^{t} b\left(x_{s}\right) d s
$$

and the deterministic time

$$
t_{x}=\sup \left\{t \geq 0: \quad x_{t} \notin \operatorname{int} J\right\} .
$$

When $H=1 / 2$, it was proved by Bouleau-Hirsch (see e.g. Theorem 6.3. in [2]) that $X_{t}$ has a density with respect to Lebesgue measure if and only if $t>t_{x}$. In particular $X_{t}$ has a density for all $t$ as soon as $\sigma\left(x_{0}\right) \neq 0$, which also follows from Hörmander's condition. Notice that Bouleau-Hirsch's result holds in a more general multidimensional context (but then $t_{x}$ is the entrance time of $X$ into the set where $\sigma$ has maximal rank, and $t_{x}$ is no more deterministic). In dimension 1 , we aim to extend this result to $\mathrm{fBm}$ of any Hurst index :

Theorem B Let $\left\{x_{t}, t \geq 0\right\},\left\{X_{t}, t \geq 0\right\}$ and $t_{x}$ be defined as above. Then $X_{t}$ has a density with respect to Lebesgue measure if and only if $t>t_{x}$.

We will need a lemma which extends Prop. 2.1.2 in [3], Chap. IV, to fBm.

Lemma 2 With the above notations,

$$
t_{x}=\inf \left\{t>0: X_{t} \notin \operatorname{int} J\right\} \quad \text { a.s. }
$$

Proof. According to (3), it is obvious that $\varphi(x, y)=y$ for all $x \in \mathbb{R}$ et $y \in J$ and then $\varphi_{y}^{\prime}(x, y)=1$ for all $x \in \mathbb{R}$ and $y \in \operatorname{int} J$. Set $\tau=\inf \left\{t>0: X_{t} \notin \operatorname{int} J\right\}$ and $T=\inf \left\{t>0: Y_{t} \notin \operatorname{int} J\right\}$. We have a.s.

- $t<T \Rightarrow \forall s \leq t: Y_{s} \in \operatorname{int} J \Rightarrow \forall s \leq t: X_{s}=\varphi\left(B_{s}^{H}, Y_{s}\right)=Y_{s} \Rightarrow \forall s \leq t: X_{s} \in \operatorname{int} J$ $\Rightarrow t \leq \tau$, which yields $T \leq \tau$.

- $t<\tau \Rightarrow \forall s \leq t: X_{s} \in \operatorname{int} J \Rightarrow \forall s \leq t: \varphi\left(B_{s}^{H}, X_{s}\right)=X_{s}=\varphi\left(B_{s}^{H}, Y_{s}\right) \Rightarrow \forall s \leq t$ : $X_{s}=Y_{s} \Rightarrow \forall s \leq t: Y_{s} \in \operatorname{int} J \Rightarrow t \leq T$. Hence $\tau \leq T$.

- $t<t_{x} \Rightarrow \forall s \leq t: x_{s} \in \operatorname{int} J \Rightarrow \forall s \leq t: x_{s}^{\prime}=b\left(x_{s}\right)=\frac{b \circ \varphi\left(B_{s}^{H}, x_{s}\right)}{\varphi_{y}^{\prime}\left(B_{s}^{H}, x_{s}\right)} \Rightarrow \forall s \leq t: x_{s}=Y_{s}$ $\Rightarrow \forall s \leq t: Y_{s} \in \operatorname{int} J \Rightarrow t \leq T$, so that $t_{x} \leq T$.

- $t<T \Rightarrow \forall s \leq t: Y_{s} \in \operatorname{int} J \Rightarrow \forall s \leq t: Y_{s}^{\prime}=\frac{b \circ \varphi\left(B_{s}^{H}, Y_{s}\right)}{\varphi_{y}^{\prime}\left(B_{s}^{H}, Y_{s}\right)}=b\left(Y_{s}\right) \Rightarrow \forall s \leq t: Y_{s}=x_{s}$ $\Rightarrow \forall s \leq t: x_{s} \in \operatorname{int} J \Rightarrow t \leq t_{x}$, whence $T \leq t_{x}$.

Finally, this proves that a.s. $t_{x}=T=\tau$, and completes the proof of the Lemma.

Proof of Theorem B. Suppose first that $t>t_{x}$. Recall that $X_{t}=\varphi\left(B_{t}^{H}, Y_{t}\right)$, where $\varphi$ is given by (3) and $Y$ is the unique solution to

$$
Y_{s}=x_{0}+\int_{0}^{s} L_{u}^{-1} b\left(X_{u}\right) d u
$$

where we set

$$
L_{u}=\varphi_{y}^{\prime}\left(B_{u}^{H}, Y_{u}\right)=\exp \left[\int_{0}^{B_{u}^{H}} \sigma^{\prime}\left(\varphi\left(z, Y_{u}\right)\right) d z\right]
$$


for every $u \geq 0$ - the second equality being an obvious consequence of (3). Notice that $L_{u}>0$ a.s. for every $u \geq 0$. We will also use the notation

$$
M_{u}=\varphi_{y y}^{\prime \prime}\left(B_{u}^{H}, Y_{u}\right)=L_{u} \int_{0}^{B_{u}^{H}} \sigma^{\prime \prime}\left(\varphi\left(z, Y_{u}\right)\right) \varphi_{y}^{\prime}\left(z, Y_{u}\right) d z
$$

the second equality coming readily from (3) as well.

We now differentiate the random variables $X_{u}, u \leq t$. Fixing $s \in[0, t]$ once and for all, the Chain Rule (see Prop. 1.2.2 in [10]) yields

$$
D_{s} X_{u}=\left(\sigma\left(X_{u}\right)+L_{u} D_{s} Y_{u}\right) \mathbf{1}_{[0, u]}(s) .
$$

In particular, setting $N_{u}=L_{u}^{-1} D_{s} X_{u}$ for every $u \leq t$, we get

$$
N_{t}=L_{t}^{-1} \sigma\left(X_{t}\right)+D_{s} Y_{t}
$$

Itô's formula (2) entails

$$
L_{t}=1+\int_{0}^{t} L_{u} \sigma^{\prime}\left(X_{u}\right) \diamond d B_{u}^{H}+\int_{0}^{t} M_{u} d Y_{u}
$$

and

$$
L_{t}^{-1} \sigma\left(X_{t}\right)=L_{s}^{-1} \sigma\left(X_{s}\right)+\int_{s}^{t}\left(\sigma^{\prime}\left(X_{u}\right)-L_{u}^{-2} M_{u} \sigma\left(X_{u}\right)\right) d Y_{u}
$$

On the other hand, differentiating $Y_{t}$ yields

$$
D_{s} Y_{t}=\int_{s}^{t} N_{u} b^{\prime}\left(X_{u}\right) d u-\int_{s}^{t}\left(\sigma^{\prime}\left(X_{u}\right)-L_{u}^{-2} M_{u} \sigma\left(X_{u}\right)+L_{u}^{-1} M_{u} N_{u}\right) d Y_{u}
$$

Putting everything together, we get

$$
N_{t}=L_{s}^{-1} \sigma\left(X_{s}\right) \exp \left[\int_{s}^{t}\left(b^{\prime}\left(X_{u}\right)-L_{u}^{-2} M_{u} b\left(X_{u}\right)\right) d u\right] .
$$

Hence,

$$
D_{s} X_{t}=\sigma\left(X_{s}\right) \exp \left[\int_{s}^{t} b^{\prime}\left(X_{u}\right) d u\right]\left(\frac{L_{t}}{L_{s}} \exp -\left[\int_{s}^{t} L_{u}^{-1} M_{u} d Y_{u}\right]\right)
$$

Notice that by Itô's formula

$$
L_{u}=\exp \left[\int_{0}^{u} \sigma^{\prime}\left(X_{v}\right) \diamond d B_{v}^{H}+\int_{0}^{u} L_{v}^{-1} M_{v} d Y_{v}\right],
$$

so that

$$
D_{s} X_{t}=\sigma\left(X_{s}\right) \exp \left[\int_{s}^{t} b^{\prime}\left(X_{u}\right) d u+\int_{0}^{t} \sigma^{\prime}\left(X_{u}\right) \diamond d B_{u}^{H}\right] .
$$


Now since $t>t_{x}$, it follows from Lemma 2 and the a.s. continuity of $s \mapsto \sigma\left(X_{s}\right)$ that the function $s \mapsto D_{s} X_{t}$ does not vanish on a subset of $[0, t]$ with positive Lebesgue measure. It is then not difficult to see that the same holds for the function $s \mapsto\left(K_{H}^{*} D . X_{t}\right)(s)$. Using (6), we obtain

$$
\left\|D . X_{t}\right\|_{\mathcal{H}}^{2}=\left(D . X_{t}, D . X_{t}\right)_{\mathcal{H}}=\int_{0}^{T}\left(K_{H}^{*} D \cdot X_{t}\right)^{2}(s) d s>0 \quad \text { a.s. }
$$

Thanks to Theorem 1 , we can conclude that $X_{t}$ has a density with respect to Lebesgue measure.

Suppose finally that $t \leq t_{x}$. Then it follows by uniqueness that $X_{t}=x_{t}$ a.s. where $x_{t}$ is deterministic, so that $X_{t}$ cannot have a density. This completes the proof of Theorem B.

\section{References}

[1] F. Baudoin and L. Coutin. Etude en temps petit des solutions d'EDS conduites par des mouvements browniens fractionnaires. To appear in C. R. Acad. Sci. Paris.

[2] N. Bouleau and F. Hirsch. Formes de Dirichlet générales et densité des variables aléatoires réelles sur l'espace de Wiener. J. Funct. Analysis 69 (2), pp. 229-259, 1986.

[3] N. Bouleau and F. Hirsch. Dirichlet Forms and Analysis on Wiener Space. De Gruyter, Berlin, 1991.

[4] L. Coutin and Z. Qian. Stochastic analysis, rough path analysis and fractional Brownian motion. Probab. Theory Related Fields 122 (1), pp. 108-140, 2002.

[5] H. Doss. Liens entre équations différentielles stochastiques et ordinaires. Ann. Inst. H. Poincaré Probab. Statist. XIII (1), pp. 99-125, 1977.

[6] D. Feyel and A. de la Pradelle. Curvilinear integrals along rough paths. Preprint Evry, 2004.

[7] M. Gradinaru, I. Nourdin, F. Russo and P. Vallois. $m$-order integrals and Itô's formula for nonsemimartingale processes; the case of a fractional Brownian motion with any Hurst index. To appear in Ann. Inst. H. Poincaré Probab. Statist.

[8] I. Nourdin. PhD Thesis, Nancy, 2004.

[9] I. Nourdin and T. Simon. On the absolute continuity of Lévy processes with drift. To appear in Annals of Probability.

[10] D. Nualart. The Malliavin Calculus and Related Topics. Springer-Verlag, 1995.

[11] D. Nualart and Y. Ouknine. Stochastic differential equations with additive fractional noise and locally unbounded drift. Preprint Barcelona, 2003.

[12] F. Russo and P. Vallois. Forward, backward and symmetric stochastic integration. Probab. Theory Rel. Fields 97 (4), pp. 403-421, 1993.

[13] H. J. Sussmann. On the gap between deterministic and stochastic ordinary differential equations. Ann. Prob. 6, pp. 19-41, 1978. 